\title{
What is moving? \\ A Special Case of Chinese Time Passing Is Motion
}

\begin{abstract}
By Shuping Huang ${ }^{*}$
This study investigates Chinese "Motion-Verb + Time" structure with an aim to identify the construal of moving entity (or entities) in this construction, and further mull over its possible implications to Chinese 'Time Passing is Motion'. Converging data from questionnaire and experiment support the argument that the omitted subject in this structure is conceivable as both a moving ego and moving time, but preferably as time. We conclude that this structure is a subcase of Moving Time, in line with Ahrens \& Huang (2002). "Moving Time" is proved to be predominant in Chinese, but specific instantiations of time metaphors require a more flexible framework to allow interactions with and coexistence of "moving ego" in the same context.
\end{abstract}

Keywords: Time Metaphor, Chinese, Moving Time, Moving Ego.

\section{Introduction}

Cross-linguistic studies have demonstrated that speakers the world over, when talking about the passage of time, envisage time as a moving entity. Time Passing is Motion (or Time is Motion) can be understood as two sets of related construals: Moving Time and Moving Ego. Manifestations of the metaphor as well as the two subsets are, nevertheless, subject to scrutiny in a relativist view.

While Moving Time and Moving Ego are both attested in Chinese, scholars disagree about a proper account of Chinese time construal. Grammatical structure, particularly clausal subject, is used as a clue to sort out what is moving - time or ego. But in a pro-drop language such as Chinese, the moving entity is often omitted, and its identity is not always clear.

In this study, special focus is placed on one such expression: "MotionVerb + Time" structure. This structure has a high frequency in corpus, but ambiguity arises over What was moving along the time axis. Previous studies fail to pin down a reading as to whether the subject (i.e. the moving entity) is the viewer (or Ego), an abstract notion of time, or an ego attached to time. Given the ubiquity of this structure, its analysis is crucial to our understanding of Chinese time construal, and we therefore launched two studies to verify different possibilities.

This paper is organized as follows. A literature review on Moving time (MT) and Moving ego (ME) is presented, followed by relevant debates and varying analyses in Chinese. A special case of subjectless structure is then examined. In two empirical studies, we attempted to explicate the temporal

\footnotetext{
${ }^{*}$ Assistant Professor, Feng Chia University, Taiwan.
} 
construal of this structure. Converging results show that such structure is conceivable as both MT and ME, but the fundamental construal should be MT. Finally, we conclude with a discussion and deliberation on a proper analysis of this structure, with implications to the Chinese construal of moving time.

\section{‘Time is Motion': Two Sub-models}

In a vast amount of literature, Time is Motion is agreed to have two instantiations, or two sub-models: Moving time and Moving ego (Clark, 1973; Lakoff \& Johnson, 1999; Gentner et al., 2002; Gentner, 2001). The Moving time (MT) model is a construal in which the speaker imagines time as moving along a temporal axis, from the past to future, and the viewer stands watching. The other is a Moving Ego (ME) model in which the viewer moves on a static temporal axis, and reaches specific temporal points. In English, for example, MT is manifested in sentences such as Time flies, or The show is drawing near. $\mathrm{ME}$, on the other hand, is illustratable as in We just stepped into a new millennium or We have entered a new age of mass marketing.

The psychological reality of these two models has been verified by experiments - results showed that the switch between the two models led to processing cost (Gentner \& Imai, 1992; McGlone \& Harding, 1998). McGlone \& Harding (1998) suggested that in ME, the ego is moving facing the future, so the resulting construal is "later time in front of earlier time." On the contrary, in MT, time is moving toward ego, or past-ward, which leads to the construal "earlier time in front of later time." An ambiguous expression like "a meeting is moved forward" is interpretable in $\mathrm{ME}$ as "being moved to a later time" while the MT construal yields an opposite interpretation that "the meeting is moved to an earlier time." One thing to be noted is that these two models are not mutually-exclusive; the two construals may co-exist in the same sentence, an idea known as "duality" in Lakoff (1993).

In different speech communities, researchers have found that the expositions of these two models can be quite diverse. In English, ME is a predominant model (Boroditsky, 2000; Gentner, 2001). Gentner et al. (2002) investigated English speakers' ability to switch between ME and MT models. The result showed that participants were faster in understanding ME- than MTbased expressions. Similarly, Boroditsky \& Ramscar (2002) examined English speakers' preferred models of moving time, and the data also showed a penchant for ME over MT. Some languages are, on the contrary, claimed to be MT-predominant, such as Malagasy (Dahl, 1995) and Chinese (Tai, 1993; Lai, 2002; Lai \& Boroditsky 2013).

\section{Debates on Chinese Time Passing is Motion}

Chinese is a thorny case. Most studies demonstrated that Chinese speakers tend to reason with MT. Lai \& Borodistky (2013), for example, followed McGlone \& Harding (1998) and asked participants to respond to questions 
such as "Next Wednesday's meeting has been moved forward two days. What day is the meeting now that it has been rescheduled?" McGlone \& Harding (1998) have already shown that English speakers tend to adopt an ME construal and answered "Friday." Lai \& Borodistky (2013) instead showed that Chinese speakers were prone to "Monday," i.e. earlier time in the front.

However, the findings in Lai (2002) and Lai \& Boroditsky (2013) may not have addressed all the controversies raised in Chinese literature. Huang \& Hsieh (2007) reported a contradicting result; Chinese speakers were faster to understand ME-based sentences when the stimuli were presented in Chinese, but faster to understand MT when the sentences were in English. Further studies have to be done to verify whether the inconsistent results came from participant idiosyncrasies.

A tendency is found in more recent studies that a simple ME-MT division cannot gracefully account for Chinese expositions of Time Passing as Motion metaphor. Yu (1998) proposes two inference patterns - Case 1 and Case 2 for Chinese Time as Space metaphors, roughly equivalent to MT and ME, and to the binary classification, he adds Case 3 in which time and observer move in the same direction over a stationary temporal landscape. Following Lakoff \& Turner (1989), Yu subsumes Case 3 under Case 2 for the reason that Case 3, just like the ME construal, requires a temporal landscape and a moving observer. Chiu (1998), while basically following his view, believes Case 3 is better classified as a general pattern for Case 1, reducing the inference patterns to two: a) Moving time with ego attached and b) Moving ego. Ahrens \& Huang (2002), however, argue convincingly that Moving time is fundamental in Chinese; when time moves, ego also moves with it. They propose a general metaphor Time Passing is Motion, which instantiates two special cases that are in essence consistent: Time Passing is a Moving Object in which time moves toward the ego, and Time Passing is a Moving Point on a Landscape in which a "time point" is moving, and ego is attached. The ego can be a collective sense for an achievement motion verb, or a singular sense for an activity motion verb. Table 1 summarizes three major studies regarding MT-ME controversy.

Table 1. Three Postulations of Chinese Time Metaphors in Comparison.

\begin{tabular}{|l|l|l|}
\hline Yu (1998) & Chiu (1998) & Ahrens \& Huang (2002) \\
\hline $\begin{array}{l}\text { Case 1: Time as moving } \\
\text { toward the face of the } \\
\text { stationary Observer }\end{array}$ & $\begin{array}{l}\text { Both time and ego are } \\
\text { moving. }\end{array}$ & $\begin{array}{l}\text { Time Passing is Motion } \\
\text { 1. Time Passing is a Moving Entity }\end{array}$ \\
\hline $\begin{array}{l}\text { Case 2: Time as bounded } \\
\text { space over which the } \\
\text { Observer is traveling }\end{array}$ & $\begin{array}{l}\text { Ego was moving and } \\
\text { time is stationary. }\end{array}$ & $\begin{array}{l}\text { 2. Time Passing is a Moving Point } \\
\text { on a Landscape (the ego is attached } \\
\text { to this point) }\end{array}$ \\
\hline $\begin{array}{l}\text { Case 3: A subcase of Case 2. } \\
\text { Time as both a moving entity } \\
\text { and a fixed location }\end{array}$ & & \\
\hline
\end{tabular}

The position held by Ahrens \& Huang (2002) is similar to Yu (1998): Moving Time is fundamental in Chinese time construal, while the motion of Ego is optionally profiled. On the contrary, Chiu's classification shows the 
belief that Ego is conceptualized as always on the move, while Time can be a moving object or a stationary landscape.

Note the when we consider which is fundamental - ME or MT, we do not claim the frequent construal as being more basic. Frequency means higher instantiations of a metaphor, but here we focus on the cognitive basis of time representation, namely, which one is logically easier to substantiate a a consistent time conceptualization in Chinese. Still, it is an unsettled debate over which framework can most gracefully and faithfully account for the Chinese time concept.

\section{A Special Case in Chinese: "Motion-Verb + Time"}

It is noteworthy that grammar is often used as a clue to the analysis of Chinese time metaphors. For example, in Ahrens \& Huang, the subject of a clause is considered an indicator to the moving entity, as Ego in (1), or as Time in (2). Generally, MT sentences are introduced by a temporal subject such as "time" or "yesterday," while ME expressions take a human subject such as "I" or "we."

(1) women yijing jinru le ershiyi shiji we already enter LE twenty-first century

'We have already entered the twenty-first century.' (Ahrens \& Huang (2002, p. 502)

(2) shengdanjie kuai dao le. Christmas quick arrive ASP

'Christmas is almost here. (Ahrens \& Huang 2002, p. 500)

Using a subject as a clue for ME or MT, has made metaphor analysis easier, but real data is not always as straightforward and clear-cut. In Chinese, the omission of subjects is frequent and natural, and some expressions therefore leave researchers who aim at a clear classification, puzzled. For example, in (3), both ME and MT interpretations are logical.

(3) Guo le shengdanjie, women haimei jianmian.

pass LE Christmas we not yet meet

'Christmas had passed (or, We had passed Christmas), yet we haven't met.'

In (3), the subject of guo 'pass' is omitted, and in Chinese the subject in this structure is regularly omitted. Consequently, it is hard to determine whether it is Time that is conceptualized as moving toward a specific point, i.e. bedtime, or whether it is Ego that is moving toward a temporal point.

Such a 'subjectless' construction is not tackled in previous studies, but its high frequency in corpus suggests that a proper analysis is non-trivial. To show 
the relative frequency of ego-subject, time-subject, and subjectless Time Motion metaphor, we investigated these three constructions in Sinica Balanced Corpus (SBC) using Sketch Engine. Co-occurrence patterns were inspected, focusing on three time phrases shijian, shihou, and shike, all meaning 'time,' with two major motion verbs dao 'arrive' and guo 'pass.' These two verbs were chosen because they are the most frequent motion verbs collocating with Time, outnumbering other motion verbs such as jinru 'enter,' hui 'return,' or lailin 'come.' Concordance was established for each time phrase with each motion verb, and the concordance was then checked manually for the three time constructions in question. The frequencies for different collocating patterns are illustrated in Table 1 below.

Table 1. The Frequency of Three Time Constructions in SBC.

\begin{tabular}{|c|c|c|c|c|c|c|}
\hline Types & Subjectless & No. & Time-subject & No. & Ego-subject & No. \\
\hline \multirow{4}{*}{$\begin{array}{l}\text { Dao-type: } \\
\text { arrive + Time }\end{array}$} & dao + shijian & 25 & shijian + dao & 65 & Ego + dao shijian & 2 \\
\hline & dao + shihou & 133 & shihou + dao & 9 & Ego + dao shihou & 10 \\
\hline & dao + shike & 18 & shike + dao & 3 & Ego + dao shike & 3 \\
\hline & & 176 & & 77 & & 15 \\
\hline \multirow{4}{*}{$\begin{array}{l}\text { Guo-type: } \\
\text { pass + Time }\end{array}$} & guo + shijian & 32 & shijian + guo & 37 & Ego + guo shijian & 1 \\
\hline & guo + shihou & 10 & shihou + guo & 0 & Ego + guo shihou & 1 \\
\hline & guo + shike & 0 & shike + guo & 0 & Ego + guo shike & 0 \\
\hline & & 42 & & 37 & & 3 \\
\hline Total & & 218 & & 114 & & 18 \\
\hline
\end{tabular}

The subjectless structure (218 tokens) is more frequent than time-subject structure (114 tokens), whereas ego-subject instances are rare (18 tokens).

An intuitive analysis is to treat "Motion-Verb + Time" structure as the Time is Space mapping of "(Subject +) Motion-Verb + Location" construction (e.g. ( $\mathrm{Ta}$ ) dao taibei le '(He) arrived at Taipei.'). As "time" now serves as a landmark in the "Motion-Verb + Time" structure, it is conceived as being static and the moving entity is presumably the viewer, or ego. This analysis contains the least change of Time is Space; only the spatial location is replaced by a temporal location. An illustration of this analysis is (4).

(4)
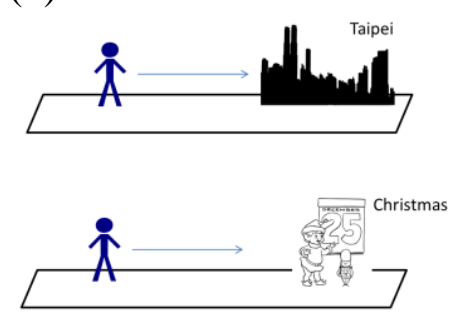

Nevertheless, a close look at the instances shows that such analysis is oversimplified. In particular, the following examples invite us to reexamine the case minutely. In some "Time + Motion-Verb" instances in the corpus, such as (5), the subject is time, and it moves past a temporal point "midnight." Again in (6), time is the subject that moves and reaches a clock time "five." 


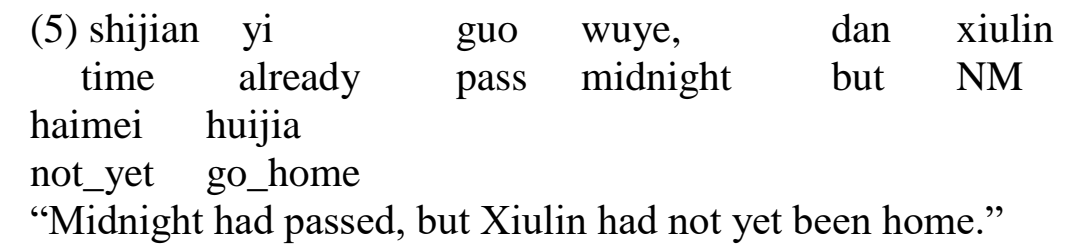
(6) shijian yi dao wudian, dajia kaishi time already arrive 5_o'clock everyone start dabao xingli pack baggage
"It's five o'clock, and everyone started to pack the baggage."

Based on these examples, it is reasonable to assume the double interpretation of "time" in Chinese: one as a moving entity, and the other as a "temporal field with fixed time points." In other words, the omitted subject of "Motion-Verb + Time" does not have to be ego or human; it can be moving time. Following Yu (1998), as reviewed earlier, this structure could be an instance of Case 3. This analysis is illustrated in (7).

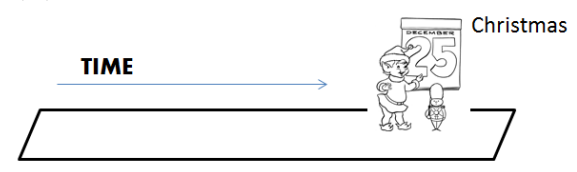

Still, (5) and (6), with time as the clausal subject, are likely to be different from a subjectless structure such as (3). And if it is correct that Ego and Time may move at the same time, according to Chiu (1998) and Ahrens \& Huang (2002), we have yet another possibility that the moving entity is both Ego and Time, although it is not clear whether MT or ME is more fundamental. This analysis is illustratable as (8).

(8)

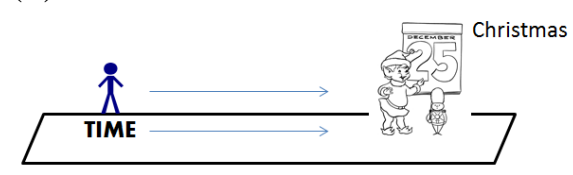

The subjectless structure accounts for such a high proportion that it certainly should not be ignored for an appropriate analysis of Chinese time metaphor - $62 \%$ (218 out of 315) of the instances in Table 1 are subjectless. Also, given that the subject is not linguistically realized, this structure can act as a good starting point for an examination of the preferred time construal in Chinese. When no subject is mentioned, what Chinese users think is moving, may serve as a clue to untie the knot of the Chinese MT-ME controversy.

To learn what is conceived as the moving entity, we launched 2 studies with special focus on this ambiguous structure "Motion-Verb + Time." The 
result can help us picture Chinese construals of time more accurately, and hence serves as a reference for future studies of language-specific time metaphors.

\section{Study 1: A Subject-Identification Questionnaire}

\section{Materials}

To resolve our doubt raised, we designed Study 1, which resorts to speaker intuition in order to learn what the speakers feel is moving in these expressions. Five ambiguous instances of "Motion-Verb + Time" were selected, together with five filler sentences, to draw up a questionnaire. Although the sentences are all perfectly acceptable without a subject, a blank was created to indicate a missing element. Please refer to Appendix 1 for these ambiguous sentences.

\section{Participants}

Thirty-three students of Sun-Yat Sen University in Taiwan were invited to complete the questionnaire. They were native Chinese speakers, 21 females and 12 males, aged from 18 to 22 . By completing this questionnaire, they could obtain two bonus points for an English course they were taking.

\section{Procedure}

Participants received a link to the online questionnaire developed by Survey Monkey. For each stimulus sentence, participants were asked to identify the missing element in the blank. Participants were given two options to choose from. For our target sentences with ambiguous reading, the two options were a time phrase and a human NP (sometimes a group). As for the fillers, the options were a non-human entity and a human NP. The order of the stimulus sentences and the two options were balanced. Participants could choose only one option, but if they were not satisfied with both options, they were allowed to provide their free response by keying in a noun.

Result

After collecting the responses from the participants, we calculated the number of time-responses, ego-responses, and irrelevant responses for each target sentence. Table 2 summarizes the result of Study 1. The following responses are analyzed as time: shijian 'time,' shidai 'time; era,' and shixu 'time course.' The following responses are analyzed as ego: renlei 'mankind,' shijie 'world,' women 'we,' huiyi 'meeting,' and guojia 'country; nation.' Irrelevant responses refer to non-NP responses such as "in the afternoon," or "based on the news." 
Table 2. Responses of the Subject-Identification Questionnaire.

\begin{tabular}{|l|l|l|l|}
\hline Test sentences & Time_Response_ & Ego_Response & Irrelevant \\
\hline Day & 17 & 14 & 2 \\
\hline Hour & 10 & 23 & 0 \\
\hline Clock time & 27 & 4 & 2 \\
\hline Season & 15 & 17 & 1 \\
\hline Century & 17 & 15 & 1 \\
\hline & $86(52.1 \%)$ & $73(44.2 \%)$ & $6(3.7 \%)$ \\
\hline
\end{tabular}

The result shows that participants, after reading the ambiguous sentences, are more likely to interpret the moving theme as "time" than as "ego," but the difference is slight, respectively 52.1 and $44.2 \%$ (3.7\% irrelevant responses are excluded).

Idiosyncrasy is also observed; for the passing of days, seasons and centuries, the preference for Time and Ego is roughly equal, but for Hour and Clock time, the preferred interpretation is not consistent. For clock time, we see a very strong MT interpretation. "Time has passed 3 o'clock" is favored over "The office has passed 3 o'clock." The use of clocks as a time-measure artifact might account for such an overwhelming preference. The fact that we can see the physical motion of hour-hands might support our conceptualization of moving time. Nevertheless, in the case of "two hours," the ME construal "The meeting has passed two hours" is preferred over "The time has passed two hours." Apparently, whether the post-verbal temporal phrase refers to a fixed time or duration does not determine speakers' interpretation. As we can see, the stimulus sentences for Day and Hour refer to duration of time, while Clock time, Season, and Year refers to passage of time past a fixed temporal location. This difference, however, does not explain speakers' preference for MT or ME. We are still not sure what has contributed to the preference for ME in the case of Hour. More examples need to be included in future studies to determine whether this result is due to the idiosyncratic effect of the stimulus sentence.

Nevertheless, a consistent tendency of all the stimulus sentences is that speakers feel the missing elements can be either Time or Ego. Responses of individual participants are examined, and none of them insist on using Time or Ego in all five target sentences. This has shown that for the subjectless time expressions, MT is slightly preferred over ME, but there is no fixed interpretation; either time or ego is moving, and it is also likely that both are moving.

\section{Study 2: Processing Experiment}

\section{Materials}

Twelve short texts with ambiguous reading were selected for the test. Each text contains two "Motion-Verb + Time" sentences. Theoretically, one 
"Motion-Verb + Time" sentence should be enough to trigger an MT or ME reading, but to increase the effect and to make the context more accessible to the participants, two "Motion-Verb + Time" sentences were used. Another eight two-sentence texts were included as fillers, which are also temporal expressions, but no metaphor was used in those filler expressions. So altogether, each participant has to read 20 texts.

Followed by each short text, participants were presented with two options - one went with the previously-shown text and the other without - and they were asked to select the logical one. For all target texts, options were presented in two construals: MT and ME. To balance the exposure to two construals, we divided the materials into two versions, each version containing six ME options and six MT options. For example, in Version 1, Text 1 is followed by ME options, and Text 12 by MT options. In Version 2, Text 1 is instead followed by MT option and Text 12 by ME option, as shown in Table 3. MT and ME options are controlled to have roughly the same number of words. Please refer to Appendix 2 for the two versions.

Table 3. Sample Stimuli for Study 2

\begin{tabular}{|c|c|c|}
\hline Setting text & Version 1 & Version 2 \\
\hline $\begin{array}{l}\text { Text } 1 \\
\text { jingguo shijiu shiji, } \\
\text { jinru ershi shiji, } \\
\text { 'X passed the } 19^{\text {th }} \\
\text { century, entered the } 20^{\text {th }} \\
\text { century,' }\end{array}$ & $\begin{array}{l}\text { Options (ME) } \\
\text { (A) renlei laidao diannao } \\
\text { shidai. 'Humans entered the } \\
\text { computer era.' } \\
\text { (B) renlei laidao shiqian } \\
\text { shidai. 'Humans entered the } \\
\text { prehistoric age.' }\end{array}$ & $\begin{array}{l}\text { Options (MT) } \\
\text { (A) diannao shidai lailin le. 'The } \\
\text { computer era arrived.' } \\
\text { (B) shiqian shidai lailin le. 'The } \\
\text { prehistoric age arrived.' }\end{array}$ \\
\hline $\begin{array}{l}\text { Text } 12 \\
\text { dao le natian banye, } \\
\text { guole liang dian, } \\
\text { 'X arrived midnight, } \\
\text { passed two o'clock,' }\end{array}$ & $\begin{array}{l}\text { Options (MT) } \\
\text { (A) ta de qingchunqi hai mei } \\
\text { dao. 'His puberty had not } \\
\text { come.' } \\
\text { (B) tade shuimianshijian hai } \\
\text { mei dao. 'His sleepy time had } \\
\text { not come.' }\end{array}$ & $\begin{array}{l}\text { Options (ME) } \\
\text { (A) ta hai mei dao qingchunqi. } \\
\text { 'He had not arrived the puberty.' } \\
\text { (B) ta hai mei dao } \\
\text { shuimianshijian. 'He had not } \\
\text { arrived the sleepy time.' }\end{array}$ \\
\hline
\end{tabular}

For the eight fillers, participants had to choose from two options that are non-metaphorical temporal expressions; one is compatible with the previous text, and the other is not. Those fillers are used in both versions.

\section{Participants}

Twelve participants were recruited to join this experiment for a pay of NT100 (approx. \$3). They were 6 males and 6 females, all native Chinese speakers, aged 18-24.

\section{Procedure}

Participants were experimented on computer screens. A two-sentence text showed at the center of the screen for 4 seconds, and then a fixation "+" appeared when participants were asked to gaze at it. When the fixation disappeared after 500 milliseconds, two options automatically showed up, and the participants were asked to choose with the keyboard an answer that went 
with the previously-shown text. When a participant selected an option, a new two-sentence text was displayed. These 20 texts were randomized, and the order of the options was balanced.

Result

Accuracy was very high irrespective of whether a text is followed by MT options or ME options. The accuracy was $95.8 \%$ for ME options, and 97.2\% for MT options. The difference is not significant $(t=0.56, \mathrm{p}>.05)$. Such high accuracy is not surprising, as the participants are asked to choose the semantically-compatible option, and the answer is apparent, based on common sense.

Nevertheless, everything being equal, a paired $t$-test shows that the same text followed by MT options was more quickly responded to than when followed by ME options $(t=2.31, \mathrm{p}<.05)$. The average RT for MT options is $1847 \mathrm{~ms}$, and $2055 \mathrm{~ms}$ for ME options. Table 4 presents the response time for each setting text.

Table 4. Response Time for Each Setting Text

\begin{tabular}{|c|c|c|}
\hline Setting text & \multicolumn{2}{|c|}{ Response time (ms) } \\
\hline 1 & ME & MT \\
\hline 2 & 1443.3 & $\mathbf{1 6 1 5 . 2}$ \\
\hline 3 & $\mathbf{1 5 6 3 . 5}$ & 1764 \\
\hline 4 & 2437 & 2177.5 \\
\hline 5 & 2266.8 & $\mathbf{2 5 9 9 . 3}$ \\
\hline 6 & $\mathbf{2 0 5 5 . 3}$ & 1629.2 \\
\hline 7 & 2165.5 & 1465.7 \\
\hline 8 & 1749.2 & 1413.7 \\
\hline 9 & 1709 & 2563.8 \\
\hline 10 & 2893.2 & 2293.8 \\
\hline 11 & 2708.3 & 1738.5 \\
\hline 12 & 1838 & 1561 \\
\hline Average & 1825.2 & 1846.9 \\
\hline
\end{tabular}

When all the text items are analyzed, most of them facilitate MT interpretation better than $\mathrm{ME}$, showing that the moving entity is basically Time. Still, two of the setting texts activate a preference for ME. For Text 2, the ME preference is slight and idiosyncratic, but this preference is consistent for Text 5, which deserves scrutiny. Let us consider Text 5 below.

(8) Setting text 5
dao le jiuyue,
arrive LE September
jinru qiutian,
enter autumn 
'X arrived September, entered autumn,'

Options (ME): Version 1

*(A) nongrenmen dao le shouhuoqi. farmers arrive LE harvest_time

'Farmers arrived at the harvest.'

(B) nongrenmen dao le dichaoqi. farmers arrive LE recession 'Farmers arrived at the depression.'

Options (MT): Version 2

*(A) shouhuoqi zhongyu daolai. harvest_time finally come 'The harvest finally arrived.'

(B) dichaoqi zhongyu daolai. recession finally come 'The depression finally arrived.'

The difference between the two options seems to be the presence of a human-subject, which serves as an extra clue for the topic of the utterance. We suggest that "recession," although not typically going with autumn, can be a plausible answer, too. The MT options take longer time because the participants are weighing the plausibility of the two options, whereas ME options provide an additional clue of "farmers" which immediately rules out "recession" because these two terms do not usually collocate.

\section{General Discussion}

We have shown that "Motion-Verb + Time" has a high frequency in Chinese Sinica Balanced Corpus. The subject is usually not linguistically realized, and both MT and ME may be compatible in the context. In Study 1, responses from Chinese native speakers demonstrate that the missing subject of "Motion-Verb + Time" can be "ego" or "time," but "time" is slightly preferred. In Study 2, participants' RT show that those ambiguous sentences, when followed by MT-based sentences, are responded to quicker, than when followed by ME construals.

Now let us go back to the question raised: What is moving? In our two studies, the preferred construal is consistently MT. In line with previous claims on the dominance of MT in Chinese (Tai, 1993; Lai, 2002; Lai \& Boroditsky 2013), our study also shows the same tendency. For this "Motion-Verb + Time" construction, the Moving ego hypothesis illustrated in (4) is ruled out, although intuitively, a static temporal location is to be traveled by an ego. This finding is crucial for studies of Chinese time metaphor, because Case 3 in Yu (1998) was proposed more than a decade ago, but this is the first time it has been attested in empirical studies. However, further to Yu's framework, we 
show that it is problematic to subsume Case 3 under Case 2, because the more fundamental construal should be MT rather than MT.

Another question arises as to whether Ego is also moving with Time in this construction. Our current studies do not provide enough clues to this question, but we can see in Study 1 that participants shift between Time and Ego, which shows "Motion-Verb + Time" is a construction that allows participants to make both interpretations. Whereas 'Moving time' is preferred, 'Moving ego' is also compatible with it and can be optionally profiled. Study 2 also does not rule out a moving ego underlying a predominant MT construal. In one of our experiment texts, it is shown that the additional information of Ego can help readers solve ambiguities. In this regard, the framework proposed by Ahrens \& Huang (2002) can best account for our data: MT and ME are compatible in one construal Time Passing is a Moving Point on a Landscape (the ego is attached to this point). MT is fundamental to this construal, and because no one can resist the change with time, a moving ego is a natural consequence of moving time. Their unifying treatment explains the predominance of moving time, and meanwhile accounts for the effortless shifts to the Moving ego.

Some questions remain unanswered. First, the contexts in which Ego is profiled are to be investigated. Second, the study of Huang \& Hsieh (2007) remains puzzling. Their finding that Chinese participants are faster in understanding ME sentences is contradictory to existing frameworks and also to our analysis. Further verification is needed. Finally, Gentner et al. (2002) pointed out that ME is easier to understand and process, as it fits in better with the human experience of moving about in space. It is evidenced in English, but not in Chinese. Are Chinese speakers prone to objective conceptualization, against a common ego-centric inclination? Does it have any cognitive or cultural implication? These are questions that are saved for our further study.

\section{Appendix 1. Sentences for Study 1}

Instruction: For each blank, choose a noun that best completes each sentence. Or enter a noun that you think makes the most sense of the sentence.

Targets (Ambiguous Sentences)

(Literal translations are provided in all cases).

[Day]__ zai guo liang tian, zhengfu jijiang
again pass two days government about_to
guanbi jichang
close airport
"In two days, the government is about to shut down
the airport."
$\begin{array}{ll}\text { (A) rizi 'days' } & \text { (B) zhengfu 'government' }\end{array}$




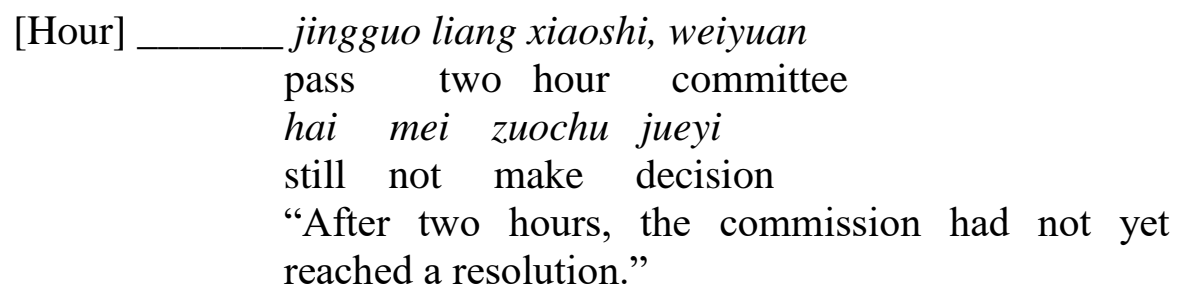
(A) huiyi 'assembly'
(B) shijian 'time'
[Clock time]__ guo le sandian, shangban de ren pass LE 3_o'clock working DE person kaishi juede pilao start feel tired

"After three o'clock, people began to feel tired."

(A) shijian 'time' (B) bangongshi 'office'

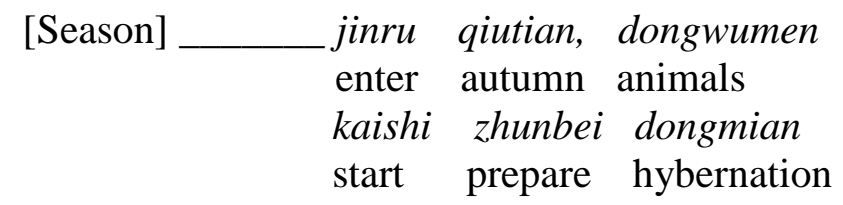

"X enters the fall, the animals began to prepare for hibernation."
(A) dadi 'the earth'
(B) shixu 'time course'

[Century] kuaru
stride_into
keji

ershiyi shiji, twenty-one century keji yuelaiyue fada technology more_and_more developed

"X strides into the 21 st century, technology is more developed."
(A) shidai 'time'
(B) renlei 'human'

\section{Appendix 2. Stimuli for Study 2 (Asterisks mark the correct answer.)}

Setting Text 1

jingguo shijiu shiji, jinru ershi shiji,
pass nineteen century enter twenty-one century
'X passed the $19^{\text {th }}$ century, entered the $20^{\text {th }}$ century,
Options (ME): Version 1
*(A) renlei laidao diannao shidai.
mankind arrive computer era
'Humans entered the computer era.'
(B) renlei laidao shiqian shidai.
mankind arrive prehistoric era


'Humans entered the prehistoric age.'

Options (MT): Version 2

*(A) diannao shidai lailin le.

computer era come CRS

'The computer era arrived.'

(B) shiqian shidai lailin le.

prehistoric era come ASP

'The prehistoric age arrived.'

Setting Text 2

dao le wushisui, kuaru gengnianqi,

arrive LE 50_years_old stride_into Menopause_period

' $\mathrm{X}$ arrived the age of 50, entered the Menopause period,'

Options (MT): Version 1

(A) nuxing de fanzhiqi daolai.

female DE breeding_time come

'Females' breeding period arrived.'

*(B) nuxing de shuaituiqi daolai.

female DE declining_period come

'Females' declining period arrived.'

Options (ME): Version 2

(A) nuxing jinru fanzhiqi.

female enter breeding_time

'Females entered the breeding period.'

*(B) nuxing jinru shuaituiqi.

female enter declining_time

'Females entered the declining period.'

Setting Text 3

dao le ershier shiji, maixiang taikong shidai,

arrive LE twenty-two century stride_to space era

' $\mathrm{X}$ arrived the $22^{\text {nd }}$ century, entered the Space Era,'

Options (ME): Version 1

*(A) renlei jinru waixing zhimin de shiqi.

mankind enter outer-space colonize DE period

'Humans entered the outer space colonial period.'

(B) renlei jinru caiji yulie de shiqi.

mankind enter gather fish_hunt DE period

'Humans entered the hunting-gathering era.' 
Options (MT): Version 2

*(A) waixing zhimin de shiqi zhongyu daolai. outer-space colonize DE period finally come 'The age of space colonization finally arrived.'

(B) caiji yulie de shiqi zhongyu daolai. gather fish_hunt DE period finally come 'The hunting-gathering era finally arrived.'

Setting Text 4

jingguo shiqi shidai, jinru qingtong shidai, pass Stone Age enter Bronze Age

'X passed the Stone Age, entered the Bronze Age,'

Options (MT): Version 1

(A) qingchao jiuci zhankai.

Qing_dynasty from_here start

'Qing Dynasty started.'

*(B) zhouchao jiuci zhankai.

Zhou_dynasty from_here start

'Zhou Dynasty started.'

Options (ME): Version 2

(A) zuxianmen jinru qingchao.

ancestors enter Qing_dynasty

'Ancestors entered the Qing Dynasty.'

*(B) zuxianmen jinru zhouchao.

ancestors enter Zhou_dynasty

'Ancestors entered the Zhou Dynasty.'

\section{Setting Text 5}

dao le jiuyue, jinru qiutian,

arrive LE September enter autumn

'X arrived September, entered autumn,'

Options (ME): Version 1

*(A) nongrenmen dao le shouhuoqi. farmers arrive LE harvest_time

'Farmers arrived at the harvest.'

(B) nongrenmen dao le dichaoqi.

farmers arrive LE recession 
'Farmers arrived at the depression.'

Options (MT): Version 2

*(A) shouhuoqi zhongyu daolai.

harvest_time finally come

'The harvest finally arrived.'

(B) dichaoqi zhongyu daolai.

recession finally come

'The depression finally arrived.'

Setting Text 6

duguo manzhang dongji, dao le meinian sanyue, pass long winter arrive LE every_year March

'X passed the long winter, arrived March every year,'

Options (MT): Version 1

(A) luoye de qiuji jiu hui lailin.

fall-leave DE autumn then will come

'Leave-falling autumn arrived.'

*(B) kaihua de chunji jiu hui lailin.

blossom DE spring then will come

'Blossom spring arrived.'

Options (ME): Version 2
(A) dadi jinru luoye de qiuji.
earth enter leave-fall DE season
'The earth entered autumns when leaves fell.'
*(B) dadi jinru kaihua de chunji.
earth enter blossom DE spring
'The earth entered spring when flowers bloomed.'

\section{Setting Text 7}

guo le shier sui, lijing xiaoxue jieduan,

pass LE twelve year go_through primary_school phase

'X passed the age of 12, went through the elementary school phase,'

Options (ME): Version 1

*(A)wo mairu guozhong qiuxue jieduan.

I stride_into junior_high study phase

'I entered the junior high school phase.'

(B) wo mairu daxue qiuxue jieduan. 
I stride_into university study phase

'I entered the university phase.'

Options (MT): Version 2

*(A) guozhong jieduan jiezhe lailin.

junior_high phase follow come

'The junior high school phase then arrived.'

(B) daxue jieduan jiezhe lailin.

university phase follow come

'The university phase then arrived.'

Setting Text 8

guo le liang nian, lijing shijici bisai,

pass LE two year go_through ten_several_time contest

'X passed the two years, went through tens of competitions,'

Options (MT): Version 1

(A) ta de huaiyunqi zongsuan jieshu le.

he DE preganant_time finally end ASP

'His pregnant period finally ended.'

*(B) ta de xunlianqi zongsuan jieshu le.

he DE training finally end ASP

'His training period finally ended.'

Options (ME): Version 2

(A) ta zongsuan auguo le huaiyunqi.

he finally survive LE pregnant_time

'He finally passed the pregnant period.'

*(B) ta zongsuan auguo le xunlianqi.

he finally survive LE training

'He finally passed the training period.'

Setting Text 9

dao le nanian dongtian, guo le eryue,

arrive LE that_year winter pass LE February

' $\mathrm{X}$ arrived the winter that year, passed February,'

Options (ME): Version 1

*(A) wo hai mei duguo shilianqi.

I still not pass break_up

'I had not got over the breakup.' 
(B) wo hai mei duguo chanluanqi.

I still not pass egg_laying

'I had not passed the egg-laying period.'

Options (MT): Version 2

*(A) wo de shilianqi hai mei guoqu.

I DE break_up still not pass

'My heart-broken time had not passed.'

(B) wo de chanluanqi hai mei guoqu.

I DE egg_laying still not pass

'My egg-laying period had not passed.'

Setting Text 10

guo le hunli zhihou,jinru xingfu shenghuo,

pass LE wedding after enter happy life

' $\mathrm{X}$ passed the wedding ceremony, entered the happy life,'

Options (MT): Version 1

(A) liangren de tongnian yanxu le liushi nian. two_person DE childhood continue LE sixty year

'Their childhood lasted for 60 years.'

*(B) liangren de aiqing yanxu le liushi nian. two_person DE love continue LE sixty year 'Their love lasted for 60 years.'

Options (ME): Version 2

(A) liangren duguo le liushi nian tongnian shenghuo. two_person pass LE sixty year childhood life 'They passed the 60-year childhood life.'

*(B) liangren duguo le liushi nian aiqing shenghuo. two_person pass LE sixty year love life 'They passed the 60-year love life.'

Setting Text 11

lijing duo nian molian, daoda biyedianli,

go_through many year tempering arrive graduate_ceremony

'X went through years of training, arrived the graduation ceremony.'

Options (ME): Version 1

*(A) ta kaixindiburu rensheng xin lucheng.

he happily step_into life new trip 
'He happily went on a journey of the new life.'

(B) ta kaixindi buru rensheng zhongdian.

he happily step_into life finish

'He happily entered the end of life.'

Options (MT): Version 2

*(A) ta yingjie rensheng xin lucheng de daolai.

he welcome life new trip DE come

'He welcome the journey of the new life.'

(B) ta yingjie rensheng zhongdian de daolai.

he welcome life finish DE come

'He welcome the end of life.'

\section{Setting Text 12}

dao le natian banye, guo le liang dian,

arrive LE that_day midnight pass LE two o'clock

' $\mathrm{X}$ arrived midnight, passed two o'clock,'

Options (MT): Version 1

(A) ta de qingchunqi hai mei dao.

he DE puberty still not arrive

'His puberty had not come.'

*(B) ta de shuimianshijian hai mei dao.

he DE sleepy_time still not arrive

'His sleepy time had not come.'

Options (ME): Version 2

(A) ta hai mei dao qingchunqi.

he still not arrive puberty

'He had not arrived the puberty.'

*(B) ta hai mei dao shuimianshijian.

he still not arrive sleepy_time

'He had not arrived the sleepy time.'

\section{Acknowledgments}

A grant for this study was received from the Taiwan Ministry of Science and Technology under the research project entitled "Time metaphors in Chinese and English and conceptualization of time zones" (NSC 102-2410-H-110-010-MY2). My special thanks goes to the Chinese Sketch Engine research team which allowed us to use the sketch engine and the Chinese database for corpus investigation. I would also like to thank the two anonymous reviewers for their constructive comments to an earlier version of this paper. All errors remain mine. 


\section{References}

Ahrens, K., \& Huang, C.-R., 2002. Time passing is motion. Language \& Linguistics, 3(3), July 2002, pp.491-519.

Alverson, H., 1994. Semantics and experience: Universal metaphors of time in English, Mandarin, Hindi, and Sesotho. Baltimore, MD: Johns Hopkins University Press.

Boroditsky, L., 2000. Metaphoric structuring: Understanding time through spatial metaphors. Cognition, 75(1), pp.1-28.

Boroditsky, L., \& Ramscar, M., 2002. The roles of body and mind in abstract thought. Psychological Science, 13(2), March 2002, pp.185-188.

Chiu, H.-H., 1998. The Metaphor Time-As-Space in Mandarin. MA thesis. Chiayi: National Chung Cheng University.

Clark, H., 1973. Space, time, semantics, and the child. In: T. E. Moore, ed. Cognitive Development and the Acquisition of Language. New York, NY: Academic, pp.27-63.

Dahl, Ø., 1995. When the future comes from behind: Malagasy and other time concepts and some consequences for communication. International Journal of Intercultural Relations, 19(2), May 1995, pp.197-209.

Gentner, D., 2001. Spatial metaphors in temporal reasoning. In: M. Gattis, ed. Spatial Schemas in Abstract Thought. Cambridge, MA: MIT Press, pp.203-222.

Gentner, D., \& Imai, M., 1992. Is the future always ahead? Evidence for systemmappings in understanding space-time metaphors. In: Proceedings of the Fourteenth Annual Meeting of the Cognitive Science Society (Indiana, United States, July 29 - August 01, 1992). Hillsdale, NJ: Lawrence Erlbaum Associates, pp.510-515.

Gentner, D., Imai, M., \& Boroditsky, L., 2002. As time goes by: Evidence for two systems in processing space $\rightarrow$ time metaphors. Language and Cognitive Processes, 17(5), September 2002, pp.537-565.

Huang, M. H.-m., \& Hsieh, S. C.-y., 2007. Time-moving metaphors and ego-moving metaphors: Which is better comprehended by Taiwanese? In: Proceedings of the 21 st Pacific Asia Conference on Language, Information and Computation (Seoul, South Korea, November 01 - 03, 2007). Seoul, South Korea: The Korean Society for Language and Information, pp.173-181.

Lai, T. V., 2002. Tests of Time in Mandarin Chinese. MA Thesis. National Taiwan University.

Lai, V. T., \& Boroditsky, L., 2013. The immediate and chronic influence of spatiotemporal metaphors on the mental representations of time in English, Mandarin, and Mandarin-English speakers. Frontiers in Psychology, 4(142), April 2013, pp.141-110.

Lakoff, G., 1993. The contemporary theory of metaphor. In: A. Ortony, ed. Metaphor and thought ( $2^{\text {nd }}$ ed., pp. 202-251). Cambridge: Cambridge University Press.

Lakoff, G., \& Johnson, M., 1999. Philosophy in the Flesh. New York, NY: Basic Books.

McGlone, M. S., \& Harding, J. L., 1998. Back (or forward?) to the future: The role of perspective in temporal language comprehension. Journal of Experimental Psychology: Learning, Memory, and Cognition, 24(5), September 1998, pp.12111223.

Tai, J. H.-Y., 1993. Conceptual structure of Chinese spatial expression. In: What We Think, What We Mean, and How We Say It: Papers from the Parasession on the Correspondence of Conceptual, Semantic and Grammatical Representations 
(Chicago, IL, United States, April 22 - 24, 1993). Chicago, IL: Chicago Linguistic Society, pp.347-362.

Yu, N., 1998. The Contemporary Theory of Metaphor: A Perspective from Chinese. Amsterdam: John Benjamin 
'... 